\title{
Perforin-Positive Leukemic Cell Infiltration in the Heart of a Patient with T-Cell Prolymphocytic Leukemia
}

\author{
Yoshinori Seko, $*, * *, * * *$, Miyuki Azuma**, Hideo Yagita**, Ko Onumura**, \\ Hisamaru HIRAI*, Ryozo NAGA * and Yoshio YAZAKI*
}

\begin{abstract}
Here we report a rare case of T-cell prolymphocytic leukemia in whom leukemic killer cells, expressing a cytolytic factor perforin, infiltrated the heart. Perforin may have directly injured myocardial cells which showed marked expression of human leukocyte antigens (HLAs) and intercellular adhesion molecule-1 (ICAM-1) as well as costimulatory molecules B7 and B70, which are ligands for CD28 expressed on T-cells. In spite of chemotherapy against leukemic cells, this autoimmune process finally caused fatal congestive heart failure.
\end{abstract}

(Internal Medicine 34: 782-784, 1995)

Key words: human leukocyte antigens (HLAs), intercellular adhesion molecule-1 (ICAM-1), costimulatory signal, cell-mediated cytotoxicity, myocardial damage

\section{Introduction}

Cardiac involvement with hematological malignant tumors such as lymphomas and leukemias is known to sometimes occur. However, in many cases, there is little clinical manifestation of cardiac dysfunction. We treated a rare case of T-cell prolymphocytic leukemia in whom leukemic killer cells infiltrated the heart and may have directly injured the myocardial cells. In spite of chemotherapy against leukemic cells, this autoimmune process finally caused fatal congestive heart failure.

\section{Case Report}

A 24-year-old man with a history of ulcerative colitis was admitted to our hospital because of a high peripheral leukocyte count and marked hepatosplenomegaly. The leukocyte count was $47,900 / \mu \mathrm{l}$ with $94 \%$ atypical lymphoid cells possessing a single nucleus. The cells expressed the phenotypes of $\mathrm{CD}^{+}$, $\mathrm{CD}^{-}, \mathrm{CD}^{+}$, and $\mathrm{CD}_{4} 5^{+}$and showed monoclonal rearrangement of $\mathrm{T}$-cell receptor $B$ chain genes. Together with morphological findings by electron microscopy, we diagnosed this case as T-cell prolymphocytic leukemia and began a combination chemotherapy. Although peripheral leukemic cell count decreased temporarily, he developed progressive congestive heart failure which was resistant to diuretics and catecholamines, and disseminated intravascular coagulation. He died of multi-organ failure 5 weeks after admission. Myocardial tissue samples were dissected from both ventricles at autopsy, and were freshly frozen in liquid nitrogen. Cryostat sections $(6-\mu \mathrm{m}$ thick) were prepared, fixed in acetone, then incubated with mouse antihuman CD3 (clone Leu-4), CD4 (Leu-3a), CD8 (Leu-2a), CD45 (HLe-1), intercellular adhesion molecule-1; ICAM-1 (RR1/1), human leukocyte antigen; HLA class I (W6/32), B7 (L310), B70 (IT2.2) (1) and rat anti-HLA class II (YE2/ $36 \mathrm{HLK})$, and rat anti-mouse perforin (P1-8), which was also shown to react with human perforin, monoclonal antibodies. The procedures for staining with an anti-perforin monoclonal antibody were as described previously $(2,3)$. The sections were sequentially incubated with biotinylated anti-mouse IgG or -rat $\mathrm{IgG}$, and with avidin-biotinylated peroxidase complex (ABCimmunoperoxidase kit, Vector Laboratories, Inc., Burlingame, CA, USA), then reacted with diaminobenzidine tetrahydrochloride. The sections were counterstained with hematoxylin and photographed.

Histological study showed that there were necrosis of cardiac myocytes, interstitial edema, and diffuse cell infiltration scattered over the myocardium. Immunohistochemical study revealed that the infiltrating cells to be $\mathrm{CD}^{+}, \mathrm{CD}^{-}, \mathrm{CD}^{+}$(Fig. 1A), and $\mathrm{CD}^{+} 5^{+}$(Fig. 1B), consistent with the phenotypes of the peripheral blood leukemic cells, and it showed that most of the infiltrating cells strongly expressed a cytolytic factor, perforin,

From *the Third Department of Internal Medicine, Faculty of Medicine, University of Tokyo, Tokyo, **the Department of Immunology, School of Medicine, Juntendo University, Tokyo and ***the Institute for Adult Diseases, Asahi Life Foundation, Tokyo

Received for publication February 14, 1995; Accepted for publication April 26, 1995

Reprint requests should be addressed to Dr. Yoshinori Seko, the Third Department of Internal Medicine, Faculty of Medicine, University of Tokyo, 7-3-1 Hongo, Bunkyo-ku, Tokyo 113 

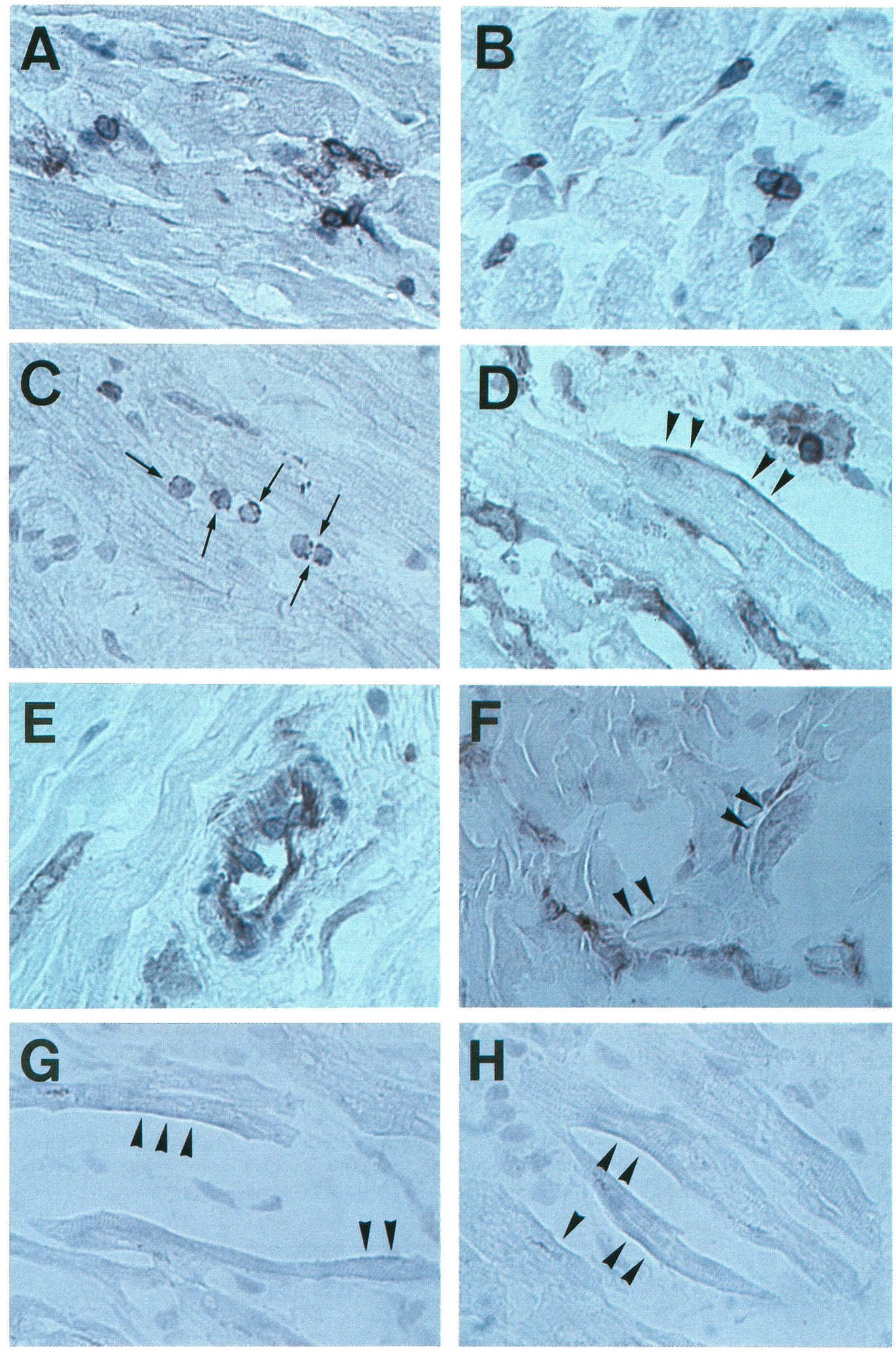

Figure 1. Immunohistochemical study of the myocardial tissue from a patient with T-cell prolymphocytic leukemia. Cryostat sections were stained with anti-CD8 (panel A), anti-CD45 (panel B), anti-perforin (panel C), anti-human leukocyte antigen (HLA) class I (panel D), anti-HLA class II (panel E), and anti-intercellular adhesion molecule-1 (ICAM-1) (panel F) monoclonal antibody. Note the clear expression of perforin in the cytoplasmic granules of the infiltrating cells (Fig. 1C, arrows) and the enhanced expression of HLA class I, ICAM-1, B7, and B70 in some of the cardiac myocytes (Figs. 1D, 1F, 1G, and 1H, respectively, arrowheads) $\times 400$. 
in their cytoplasmic granules (Fig. 1C, arrows). Recent studies (2-6) have demonstrated that perforin is expressed by infiltrating lymphocytes in various diseases, as well as by lymphocytes under physiological conditions, and can be a good marker for killer cells. This indicates that the infiltrating cells were leukemic killer cells possessing the phenotypes characteristic of cytotoxic T lymphocytes. We also found that there was enhanced expression of HLA class I, class II and ICAM- 1 in myocardial cells such as vascular endothelial cells and fibroblasts (Figs. 1D to $1 \mathrm{~F}$, respectively). Furthermore, enhanced expression of HLA class I and ICAM-1 as well as B7 and B70 was found in some of the cardiac myocytes (Figs. 1D, 1F, 1G, and 1H, respectively, arrowheads).

\section{Discussion}

Perforin, also called pore-forming protein or cytolysin, is thought to be one of the major effector molecules involved in cell-mediated cytotoxicity. It is known that perforin is released from the cytoplasmic granules of killer cells during target cell lysis, followed by its insertion and polymerization in the target cell membrane to form transmembrane tubular lesions (perforin pores), which in turn cause colloid osmotic injury of the target cells (4). In general, B7 is expressed on activated B-cells, macrophages, and dendritic cells, and is a ligand for CD28 expressed on T-cells. B70 has been identified as a second ligand for CD28, and is expressed on monocytes, dendritic cells, activated T-cells, B-cells, and natural killer cells (1). Through interaction with CD28, both B7 and B70 play an important role in potent costimulation of T-cell activation in immune responses.

T-cell prolymphocytic leukemia is a distinct clinicopathologic entity with an aggressive course and is extremely rare in Japan. Furthermore, it is known that most T-cell prolymphocytic leukemia are $\mathrm{CD} 4^{+} \mathrm{CD} 8^{-}$, a minority coexpress $\mathrm{CD} 4$ and $\mathrm{CD} 8$, and only a few are $\mathrm{CD} 4^{-} \mathrm{CD} 8^{+}$like this case (7). In the present case, it was strongly suggested that the infiltrating leukemic cells recognized cardiac myocytes as target cells and directly damaged them by releasing perforin. It is thought that the infiltrating leukemic cells recognized some antigens, probably autoantigen, presented by HLA molecules on the surface of cardiac myocytes, and that ICAM-1 as well as B7 and B70 played an important role in the recognition, adhesion, and cytotoxicity of the leukemic cells. We previously reported that in murine viral myocarditis natural killer cells infiltrate the heart and directly damage cardiac myocytes, which expressed major histocompatibility complex class I antigen and ICAM-1, by releasing perforin $(3,8-10)$. Although the immunological mechanisms involved in the myocardial damage revealed in the present case were apparently similar to those in viral myocarditis, the reasons why leukemic killer cells have lost their self tolerance against myocardial cells and why HLA and ICAM-1 as well as B7 and B70 were strongly induced on myocardial cells are still unknown and remain to be clarified.

This work was supported by a grant for cardiomyopathy and a grant for intractable vasculitis from the Ministry of Health and Welfare, Japan, a grant for scientific research from the Ministry of Education, Science and Culture, Japan, a grant from Kowa Life Science Foundation, a grant from Ichiro Kanehara Foundation, a grant from Kanae Foundation of Research for New Medicine, and a grant from Japan Foundation of Cardiovascular Research.

\section{References}

1) Azuma M, Ito D, Yagita $H$, et al. B70 antigen is a second ligand for CTLA4 and CD28. Nature 366: 76, 1993.

2) Kawasaki A, Shinkai Y, Kuwana Y, et al. Perforin, a pore forming protein detectable by monoclonal antibodies, is a functional marker for killer cells. Int Immunol 2: 677, 1990.

3) Seko Y, Shinkai Y, Kawasaki A, et al. Expression of perforin in infiltrating cells in murine hearts with acute myocarditis caused by Coxsackie virus B3. Circulation 84: 788, 1991.

4) Young JDE. Killing of target cells by lymphocytes: A mechanistic view. Physiol Rev 69: 250, 1989.

5) Young LHY, Peterson LB, Wicker LS, Persechini PM, Young JDE. In vivo expression of perforin by $\mathrm{CD}^{+}$lymphocytes in autoimmune disease: studies on spontaneous and adoptively transferred diabetes in nonobese diabetic mice. J Immunol 143: 3994, 1989.

6) Kawasaki A, Shinkai Y, Yagita H, Okumura K. Expression of perforin in murine natural killer cells and cytotoxic T lymphocytes in vivo. Eur J Immunol 22: 1215, 1992.

7) Matutes E, Brito-Babapulle V, Swansbury J, et al. Clinical and laboratory features of 78 cases of T-prolymphocytic leukemia. Blood 78: 3269, 1991.

8) Seko Y, Tsuchimochi H, Nakamura T, et al. Expression of major histocompatibility complex class I antigen in murine ventricular myocytes infected with Coxsackie virus B3. Circ Res 67: 360, 1990.

9) Seko Y, Matsuda H, Kato K, Hashimoto Y, Okumura K, Yazaki Y. Expression of intercellular adhesion molecule-1 in murine hearts with acute myocarditis caused by Cox́sackie virus B3. J Clin Invest 91: 1327, 1993.

10) Seko Y, Shinkai Y, Kawasaki A, Yagita H, Okumura K, Yazaki Y. Evidence of perforin-mediated cardiac myocyte injury in acute murine myocarditis caused by Coxsackie virus B3. J Pathol 170: 53, 1993. 